$16^{\text {th }}$ International Congress of Metrology, 09001 (2013)

DOI: $10.1051 / \mathrm{metrolog} / 201309001$

(c) Owned by the authors, published by EDP Sciences, 2013

\title{
Low cost power and flow rates measurements for manufacturing plants
}

\author{
Anne Grau ${ }^{1, a}$, Gilbert Schmitt ${ }^{1}$, Frédéric Lecoche ${ }^{2}$, Lionel Duvillaret ${ }^{2}$, Gwenael Gaborit ${ }^{2}$, Menad Bourkeb ${ }^{3}$, Charles \\ Joubert $^{3}$, Olivier Ondel ${ }^{3}$, Hamed Yahoui ${ }^{3}$, Riccardo Scorretti ${ }^{3}$, Laurent Morel ${ }^{3}$, Baya Hadid ${ }^{4}$, Régis Ouvrard ${ }^{4}$, Thierry \\ Poinot $^{4}$, Erik Etien ${ }^{4}$, and Laurent Le Brusquet ${ }^{5}$ \\ ${ }^{1}$ EDF R\&D, Dépt. EPI, E25, Centre des Renardières, 77818 Moret sur loing, France \\ ${ }^{2}$ KAPTEOS, Savoie Technolac - bât. Alouette II, 23 avenue du Lac Léman, BP 347, 73377 Le Bourget-du-Lac Cedex, \\ France \\ ${ }^{3}$ AMPERE Laboratory, Bât. Oméga, Université Lyon 1, 43 boulevard du 11 novembre, 69622 Villeurbanne Cedex, France \\ ${ }^{4}$ LIAS, Bâtiment B25, 2, Rue Pierre Brousse - BP 633, Poitiers Cedex, France \\ ${ }^{5}$ Supélec Sciences des Systèmes, Plateau de Moulon, 3, rue Joliot-Curie, 91192 Gif-sur-Yvette, France
}

\begin{abstract}
The ability to measure, monitor and control energy consumption at several key locations in a manufacturing plant is a major prerequisite for any efficient energy management program. To identify and evaluate energy savings, one must get a clear view of how the energy is used. Furthermore, measuring energy flows is one of the necessary conditions for long lasting energyefficient solutions. Most of the time energy managers are reluctant to put in place power and flow rate measuring devices either because of their cost or because this implies stopping production. To find acceptable and economical solutions for long lasting energy measurements in Industry, EDF R\&D launched a 3-year collaborative research project called CHIC. This project is funded by the French National Research Agency (ANR) and involves 7 partners. Its total budget amounts to 2.55 $M €$. This project serves two purposes: to build a clamp-on power meter that could be installed around multi-conductors power cables without interrupting power supply, and to build power and flow meters that derive the sought-for variable from mathematical models and from simple and easy to collect other physical measurements (e.g. command signals, etc...).
\end{abstract}

\section{Introduction}

In France, the energy consumption of the industrial sector amounts to $470 \mathrm{TWh}(70 \%$ of which being devoted to satisfy heating requirements). This represents roughly $28 \%$ of the French energy consumption and $23 \%$ of its total $\mathrm{CO} 2$ emissions (30 Mt/year). Part of this energy could have been saved without disrupting production nor breeching any safety rule nor impacting operators working environment. Part of this energy was wasted because it was used for purposes unnecessary for production and nobody noticed.

Nobody noticed that energy was wasted because nobody bothered to measure properly the Energy Efficiency (EE) of the equipments that were used.

\section{Implementing Energy Efficiency improvement programs}

There exists a strong potential for energy savings within the French manufacturing industry, probably equally in the European one. This potential is yet to be revealed and exploited.

Nowadays, more and more Industries are willing to save energy and therefore are implementing Energy Efficiency improvement programs. Most of such programs rely on national or international standards.

The best tools available nowadays are: the international ISO 50001 "Energy Management Systems" standard and the International Performance Measurement and Verification Protocol (IPMVP). Both rely on the proper measurement of key EE indicators.

\subsection{ISO 50001 standard for Energy Management Systems}

The ISO 50001 standard was published in June 2011. It is the result of a collaborative effort of 61 countries,

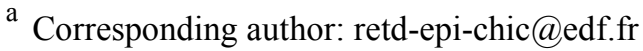


including countries from the European Committee for Standardization.

The ISO 50001 standard specifies requirements for an energy management system that is based on a continuous improvement principle Plan - Do - Check Act applied as a cycle:

- Plan: determine the main energy consuming systems and establish performance targets for it,

- Do: install a metering and monitoring system,

- Check: compare the measured energy performance to the targeted one,

- Act: identify corrective actions and implement Energy Efficiency improvement programs.

The ISO 50001 standard relies on a preliminary energy audit to determine the main energy consuming systems of the plant, and then requires setting performance targets for those systems and implementing metering and monitoring devices to check that these performance targets are respected.

\subsection{The International Performance Measurement and Verification Protocol (IPMVP)}

The International Performance Measurement and Verification Protocol was first released in 1996 and has evolved ever since. It is free to download from the Efficiency Valuation Organization (EVO) web site (http://www.evo-world.org/). EVO is a non-profit organization "dedicated to creating Measurement and Verification tools to allow efficiency to flourish".

This protocol presents a framework and defines the terms that are to be used for determining the savings one should expect after implementing an Energy Efficiency improvement program.

IPMVP focuses on three major issues which are: defining Performance, Performance Measurement and Performance Verification.

Defining Performance is a prerequisite. Performance can be defined at the plant level or at an intermediate level according to the Energy Efficiency improvement program that is to be implemented. For instance, if a compressed air system is to be refurbished, then the protocol can only focus on that specific compressed air system.

Performance Measurement requires installing measuring devices wherever needed, which depends on the Performance Verification protocol that will be used.

Performance Verification is the trickiest part of the protocol, since it is impossible to measure energy savings per se. Only energy consumption can be measured. It has to be compared to forecasted energy consumption in order to estimate how much energy has been saved. According to the protocol, the forecasted energy consumption will be calculated using a baseline / reference energy consumption and several adjustment factors that have to be defined. Typical adjustment factors would be the production load factor, the outside temperature, etc...

\subsection{Measuring is the key for improving Energy Efficiency in the manufacturing sector}

Whatever the industrial sector considered (food, cement, metal ...), the optimisation of a manufacturing plant is a complex process that requires monitoring. One cannot optimize what he / she does not know. To identify and evaluate energy savings, one must get a clear view of how the energy is used. As stated in the ISO 50001 standard, measuring is the first step towards energy consumption awareness and thereafter Energy Efficiency. The ability to measure, monitor and control energy consumption at several key locations in a manufacturing plant is a major prerequisite for any efficient energy management program.

Furthermore, all manufacturing plants are continuously evolving and what was optimized at one moment may not stay optimized for a long time. Once more, measuring is the key to maintain Energy Efficiency throughout time. Energy savings programs, when their impacts are not continuously measured, prove themselves inefficient in the long - or even short - term. Usually several months is a period of time long enough to get into a non optimized situation again. Therefore, continuously measuring energy flows is one of the necessary conditions for long lasting energy-efficient solutions.

N.B. In the manufacturing industry, two different types of energy consumption must be distinguished: the one related to the process itself and the one related to the systems that deliver compressed air, vapour, cold water, etc... through the plant. Whereas it is generally very difficult to modify the energy consumption related to a manufacturing process, because this might have a strong impact on production, it is most of the time much easier to optimize auxiliary energy consumption, as long as it is well known and understood and therefore well measured.

\subsection{Cost-benefit analysis for Energy Efficiency improvement programs}

Within the industrial sector, every investment program, and especially an Energy Efficiency improvement one, is or is not implemented according to its cost-benefit analysis. Unfortunately, most of the time, the implementation of an Energy Efficiency improvement program, because of its mandatory measurement phase, is seen as not acceptable.

Several values need to be measured within an Energy Efficiency improvement program. Some physical parameters, such as temperatures, are easy and not expensive to measure. On the contrary, power and flow rates are either rather expensive or totally impossible to measure, especially if the plant is already in operation.

To measure power for instance, one must cut the power off, for safety reasons, which generally disrupts production. To measure flow rates, one can use some regular flow meters which installation requires cutting through the pipes. This once again generally disrupts production. For many manufacturing plants, it is not acceptable to stop production to install measuring devices.

What penalizes measurements is not technology. It is costs. IPMVP suggests an additional cost for measuring of less than 10 to $15 \%$ of the program total 
energy savings. What penalizes measurements is the additional cost of disrupting production during the installation of the meters, which is most of the time way above the recommended and acceptable 10 to $15 \%$.

\section{The CHIC research project}

CHIC is a French acronym for "CHaîne de mesures Innovantes à bas Coût", which means: innovative low cost measuring systems.

The CHIC research project was launched in January of 2010 in order to tackle measuring issues in manufacturing plants. It is a 3-year project funded by the French National Research Agency (ANR). It involves 7 partners: EDF R\&D, AMPERE, LIAS, CEA-LETI, Kapteos, SOCOMEC and SUPELEC. The total budget of the project amounts to $2.55 \mathrm{M} €$.

As stated above, not every measurement is problematic. The CHIC research project focuses on:

- power measurements for three-conductor power cables,

- flow rate measurements.

N.B. These measurements are the most popular ones within the energy plant manager community, which are really eager to get such values, and the most unpopular ones within the plant manager community because of their installation consequences and costs.

The CHIC research project focuses on creating and experimenting new solutions that will be:

- non intrusive,

- low cost,

- plug and play,

- low energy consumption systems,

- efficient and robust, even with noise and perturbations.

New measuring devices are being developed during the CHIC research project. Some of them are "software based meters" - as opposed to "physical meters" -, because they derive their measurement from mathematical models and from simple and easily measurable other physical variables (like command signals for instance).

The following meters will be developed during the $\mathrm{CHIC}$ project (see later for details):

- a physical clamp-on power meter that could be installed around three-conductor electrical cables anywhere in the plant,

- a software power meter, for industrial electrical furnaces, that will derive power from the furnace command signal,

- a software compressed air flow meter, that will derive the air flow rate from the compressor consumed power,

- a software gas flow meter for boilers, that will derive the gas flow rate from its inlet valve opening position.

N.B. Every CHIC software meter must be dedicated to a specific equipment because it relies on extra variables and on mathematical models that are strongly dependant on the physics involved.

The four different prototypes realized within the project will be tested on EDF R\&D premises so as to validate their use in manufacturing plants. Three EDF R\&D laboratories will be used for the tests, each of them being dedicated to equipments that can be found in
Industry: chillers, electrical furnaces and gas boilers. CHIC measuring devices will be tested on a $75 \mathrm{~kW}$ chiller, on a $146 \mathrm{~kW}$ electrical furnace and on a $750 \mathrm{~kW}$ gas boiler. The EDF R\&D research Centre of Les Renardières near Paris has also a dedicated compressed air station, which supplies EDF R\&D laboratories. This compressed air station will also be used for the tests.

The facilities used to test the project prototypes are similar to those found in most French manufacturing plants. They may be a little less powerful, but they will allow testing CHIC measuring devices in real and industry-like operating situations (with noise, perturbations etc...).

\section{State of the art for power and flow rate measurements}

Methodologies and costs for power and flow rates measurement with actual commercial devices were investigated at the beginning of the project. Our main foundings are presented below.

\subsection{Measuring power for three-conductor power cables with actual commercial devices}

\subsubsection{Methodology used}

Nowadays there exist a lot of commercial devices that can be used to measure power for three-conductor power cables. They all use the same methodology.

Today's energy meters need to calculate power from the conductors current and voltage values, therefore the need to measure three currents and three voltages. This can only be done inside an electrical distribution box, where the conductors of the power cable are separated. Voltages are measured with probes directly connected to the conductors. Currents are measured by installing around each conductor a current transformer. Such devices reproduce the current that flows through a conductor at a much lower scale. Examples of devices that can be used to measure currents in one conductor are given in [1]. Measuring power for a three-conductor power cable means installing three voltage probes and three current transformers inside the electrical distribution box and connecting all six to an electrical energy meter (see below for a schematic description of power measurement with actual commercial devices). For safety reasons, it is mandatory to cut power when installing the current transformers and the voltage probes inside the electrical distribution box.

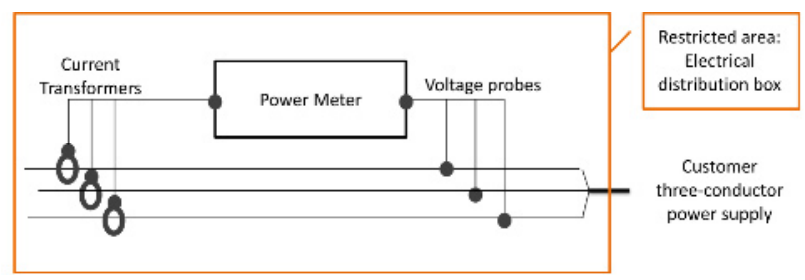

Figure 1. Measuring power with today commercial metering devices. 
The main disadvantages of this solution are:

- for safety reasons, power must be cut off to install all voltage probes and current transformers, which means stopping all electrical devices that are connected to the distribution box where the meter is installed,

- only authorized and specifically trained technicians can open an electrical distribution box, which means that the labour costs of the measurement are relatively high.

These two major disadvantages induce most of the time high rejection rates.

\subsubsection{Costs}

One of the first tasks of the CHIC project was to quantify the cost of power measurements with actual commercial energy meters over a 20 years period of exploitation (such a long period have been chosen because it matches the exploitation expectancy of the meters). The calculations were based on typical electrical installations often found in French manufacturing plants. Those include 8 levels of current (from $60 \mathrm{~A}$ to $1500 \mathrm{~A}$ ), each of them being monitored. The total cost of the monitoring system that should have been put in place on those typical electrical installations has been calculated and then divided by 8 to retrieve the average cost for one power measurement. Two types of current transformers were used for the calculations: one of them being a standard one, and the other one, more expensive, being designed for retrofit operations because it can be opened (its installation is therefore easier but nevertheless requires cutting off power). The results are showed in the table below:

Table 1. Average costs for power measurement for one threeconductor electrical cable with today commercial meters over a 20 years exploitation period (no interest rate).

\begin{tabular}{|l|l|l|}
\hline & $\begin{array}{l}\text { Standard energy } \\
\text { meter }\end{array}$ & $\begin{array}{l}\text { Retrofit energy } \\
\text { meter }\end{array}$ \\
\hline $\begin{array}{l}\text { Average costs (sum } \\
\text { of purchase, } \\
\text { installation and } \\
\text { maintenance costs) }\end{array}$ & $1409 €$ & $1726 €$ \\
\hline $\begin{array}{l}\text { Total amount of } \\
\text { time during which } \\
\text { power must be cut } \\
\text { off to provide safety } \\
\text { for the electrician } \\
\text { who installs the } \\
\text { meter. }\end{array}$ & $96 \mathrm{~h}$ & $32 \mathrm{~h}$ \\
\hline
\end{tabular}

N.B. All actual technologies require cutting power during the installation of the current transformers and the voltage probes, for safety reasons, which is for most Industries, not acceptable.

\subsection{Measuring flow rates with actual commercial devices}

\subsubsection{Methodology used}

Two types of actual commercial flow meters were evaluated at the beginning of the CHIC project:

- a standard electromagnetic flow meter that is very common in manufacturing plants (see figure below), and that needs cutting the pipe to be installed (the same evaluation could have been done with other types of intrusive flow meters (Coriolis, Vortex, etc...) - whatever the technology used, the results would be similar),

- a non intrusive flow meter that is based on ultrasound technology, which installation does not need cutting the pipe (see figure below). Taking the meter off the pipe does not require cutting the pipe either.
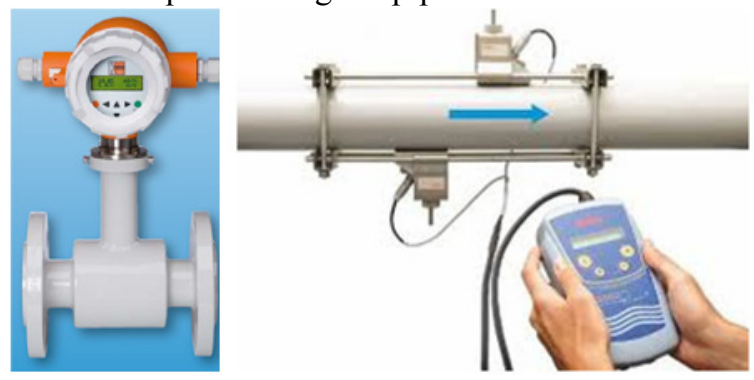

Figure 2. On the left: standard commercial electromagnetic flow meter; on the right: measuring flow rates with an ultrasound flow meter.

\subsubsection{Costs}

The total costs for flow rates measurement with these two commercial devices have been evaluated for an exploitation period of 10 years. The assumptions for the pipe and operating conditions were as follows: the diameter of the pipe was of $80 \mathrm{~mm}$, the fluid flowing in the pipe was water, its pressure was below 10 bars, its temperature was comprised between $60^{\circ} \mathrm{C}$ and $80^{\circ} \mathrm{C}$, the speed of the water was of about $7 \mathrm{~m} / \mathrm{s}$. The assumptions for the maintenance of the meters were as follows: the standard electromagnetic flow meter needs maintenance every year, which requires emptying the pipe and sending the meter for checking (the total labour costs for taking the meter off the pipe and putting it back again on the pipe is of at least $2 \mathrm{~h}$ per year), the ultrasound meter needs maintenance every 5 years, which requires sending the meter for checking (the total labour costs for taking the meter off the pipe and putting it back again on the pipe is on average of 15 min per year). The results of this analysis are showed in the table below.

Table 2. Total costs for one flow rate measurement with today commercial products over a 10 years exploitation period (no interest rate).

\begin{tabular}{|l|l|l|}
\hline & $\begin{array}{l}\text { Standard } \\
\text { electromagnetic } \\
\text { flow meter }\end{array}$ & $\begin{array}{l}\text { Ultra sound flow } \\
\text { meter }\end{array}$ \\
\hline $\begin{array}{l}\text { Purchase cost for } \\
\text { one meter }\end{array}$ & $500 €$ & $5000 €$ \\
\hline $\begin{array}{l}\text { Total costs (sum of } \\
\text { purchase, } \\
\text { installation and } \\
\text { maintenance } \\
\text { costs) }\end{array}$ & $6540 €$ & $5845 €$ \\
\hline Number of times & 10 & 0 \\
\hline
\end{tabular}




\begin{tabular}{l|l|l|} 
for which the pipe & & \\
must be emptied & & \\
and the process & & \\
stopped during the & & \\
10 years period. & & \\
\hline
\end{tabular}

N.B. Although intrusive flow meters purchase costs are very low (there is a factor of 10 between the purchase costs of the two flow meters presented above), their exploitation costs are, over a 10 years exploitation period, at the same level as those of non intrusive flow meters. Nevertheless, ultrasound flow meters are seen as very expensive and are mostly dedicated to time-limited energy audits.

Plant managers are usually reluctant to install flow rate meters on existing and operational pipes. Different reasons explain this attitude, according to the type of flow meter:

- non intrusive flow meters are seen as too expensive,

- all other flow meters, which are intrusive, require emptying and cutting the pipe to be installed.

\section{CHIC power meters}

\subsection{Costs and benefits for CHIC power meters}

The targeted total exploitation costs for CHIC power meters over a 20 years period has been set to between $1409 €$ and $1726 €$, which is the sum of the actual purchase, installation and maintenance costs for existing commercial meters over a 20 years exploitation period.

The main advantage of CHIC power meters over the actual commercial meters will be that there will be no need to cut power to install them.

Furthermore, the following advantage is foreseen for CHIC power meters: the CHIC physical clamp-on power meter will be able to support some installation checking process because of its embedded calculations: it should be able to tell if it is correctly installed. As for the CHIC software power meter, it will obviously be installed correctly (very little mistakes can be made in getting a command signal). This would be a real technological breakthrough because, as we have seen on many manufacturing plants, roughly $30 \%$ of the installed power meters do not deliver correct values, as they are not installed properly. It takes time and efforts to make sure that the installed power meters are trustworthy. This won't be the case with CHIC power meters.

\subsection{CHIC physical clamp-on power meter}

As stated above there is a need for non intrusive power meters for three-conductor power cables, i.e. for power sensors that does not require having the conductors separated nor being installed inside an electrical distribution box. It was decided to build within the CHIC project a clamp-on power meter that would derive power from the measurement of the currents and voltages of the three conductors and that will be installed directly on the three-conductor power cables, wherever these are in the plant (and especially not in restricted places such as electrical distribution boxes).

Conductors' currents will be calculated from the measurement of the magnetic field that surrounds the power cable. Similarly, voltages will be calculated from the measurement of the electrical field that surrounds the power cable. Both calculations depend on the position of the conductors inside the cable. The following figure shows the inside geometry of a typical industrial threeconductor power cable:
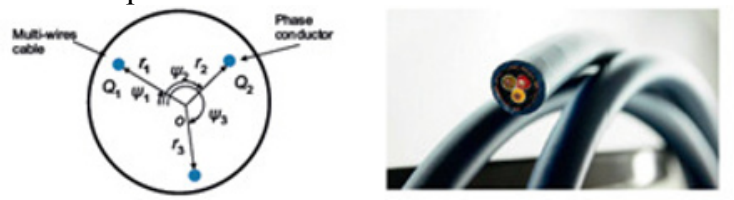

Figure 3. Cross section geometry of a typical industrial threeconductor power cable

\subsection{CHIC physical clamp-on Intensity sensor}

A bibliographical survey carried on electrical and magnetic field measurements around power cables pointed out that the $\mathrm{CHIC}$ project was facing real methodological and technical challenges in that domain [2], [3], [4].

Many simulations were conducted in order to derive a robust methodology that could be used to derive the conductors' currents and voltages from the measurement of the magnetic and electrical fields around the power cable.

It is foreseen that the CHIC physical currents sensor will look as shown in the following figure:

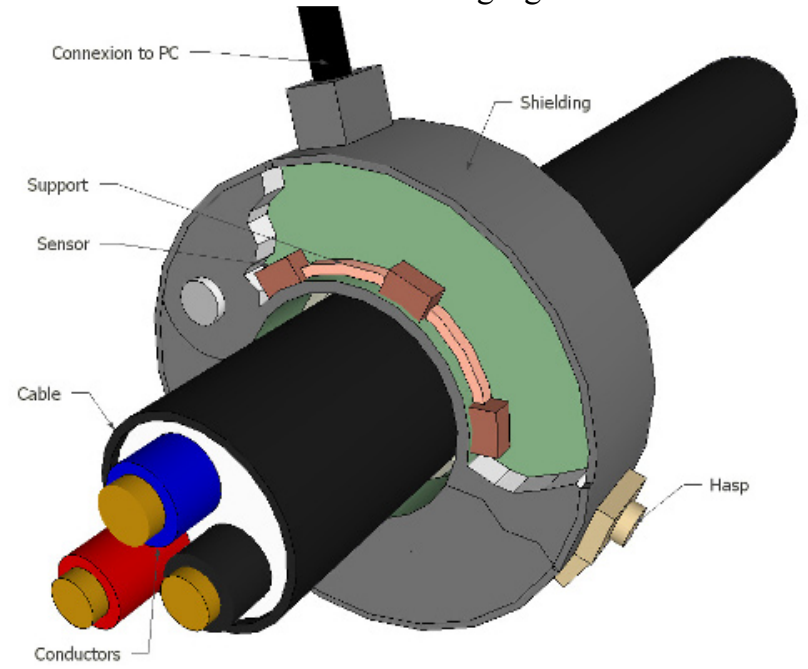

Figure 4. Design of the currents measurement device

In theory, the relationship between the magnetic field B around the power cable and the currents I flowing through the conductors is linear, therefore the possibility to derive the values of the currents from the measurement of the magnetic field distribution. Theoretical work has been carried out for different distributions of magnetic sensors (magnetometers) around multi conductor cables. $\mathrm{N}$ magnetometers were considered at first. It became soon obvious that the magnetometers around the cable should be shielded from the influence of other cables. 
The figure below represents the configuration that has been studied.

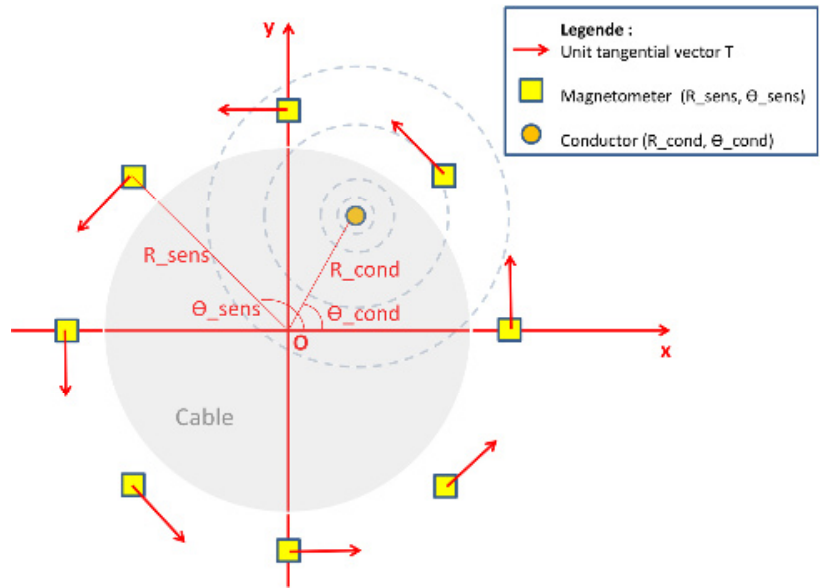

Figure 5. Cross section of the CHIC sensor positioned around the three-conductor power cable (N.B. only one conductor is represented)

The mathematical relation between the magnetic field measured in the $\mathrm{N}$ magnetometers around the cable and the values of the currents is (when considering $M$ conductors):

$$
\left(\begin{array}{c}
B_{1} \\
\vdots \\
B_{N}
\end{array}\right)=\left(\begin{array}{ccc}
k_{11} & \cdots & k_{1 M} \\
\vdots & \ddots & \vdots \\
k_{N 1} & \cdots & k_{N M}
\end{array}\right) \cdot\left(\begin{array}{c}
I_{1} \\
\vdots \\
I_{M}
\end{array}\right)
$$

with:

- $k_{i j}$ : parameters that define the system to be measured $\left(R \_\right.$sens, $R \_$cond, ...) with $\mathrm{i} \in\{1, \ldots$ $, \mathrm{N}\}$ and $\mathrm{j} \in\{1, \ldots, \mathrm{M}\}$

- $B_{\mathrm{i}}$ : flux density measured by the $\mathrm{i}$-th magnetometer.

As a consequence, the estimated currents I flowing through the conductors can be computed by solving equation ( 2 ) in the sense of least squares:

$$
\{I\}=[K]^{+} \cdot\{B\}
$$

The methodology presented above involves solving a non trivial inverse problem, with difficulties related to the unknown characteristics of the cable: its internal structure (distance between the conductors and the centre of the cable, conductors' radii, permittivity of the insulating material) and its actual position inside the ring of magnetometers. A more detailed description of the work done within the $\mathrm{CHIC}$ research project can be found in [5], [6], [7].

Although a solid theoretical background has been set for the physical CHIC power meter, much work remains to be done concerning its practical implementation. Its future geometry and its constitutive materials must be defined. A first prototype was build at the end of 2012. It has been tested in a dedicated test bench (see picture below).

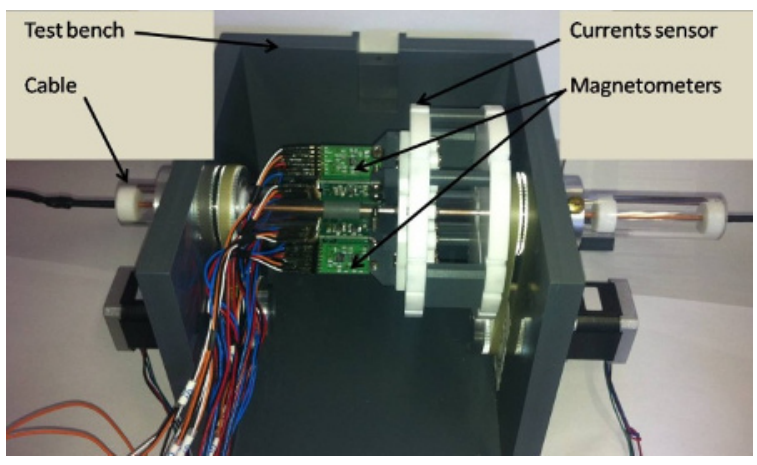

Figure 6. Test bench for the CHIC physical current sensor (tested here with no shield).

Good experimental results were obtained with no shield and without any perturbations in the measuring environment.

The following figure shows the CHIC physical current sensor within the test bench, after a shield was added around it:

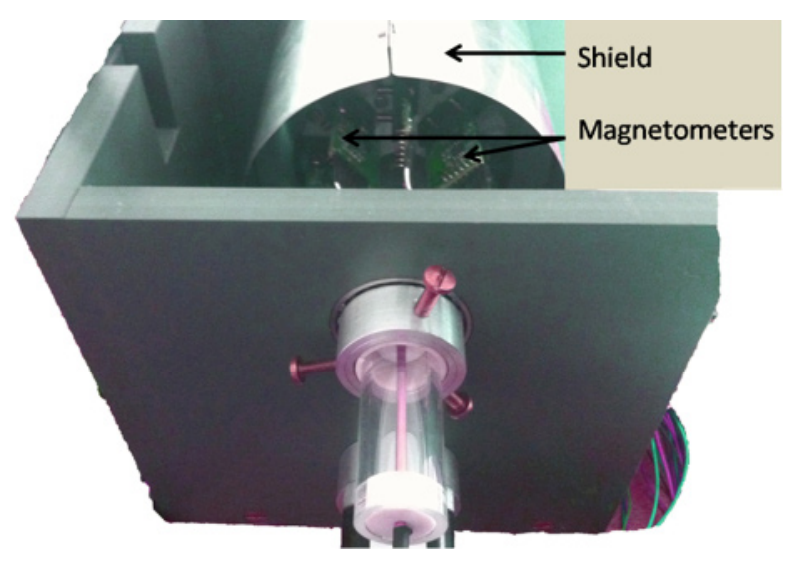

Figure 7. Testing of the shielded CHIC physical current sensor.

A specific reconstruction algorithm was applied in order to derive the values of the currents flowing through the 3 conductors from the magnetic field measurement. The entire methodology is described in [8]. It relies on a lookup table that was initially computed with a Finite Element software.

The results we got so far are very interesting and promising for an innovative sensor within a near future

This prototype is now to be tested in one of EDF R\&D laboratories, where the power delivered through the 3 conductor cable can be varied from $10 \mathrm{~kW}$ to $58 \mathrm{~kW}$.

\subsection{CHIC physical clamp-on Voltage sensor}

Concerning the voltage sensor, the contact-less measurement is ensured thanks to an electro-optic (EO) technique. A non-centrosymetric crystal sees its eigen refractive indices linearly modified by the ambient electric (E)-field: this is the Pockels effect. Based on this principle, the realized device is presented in figure 8 . 


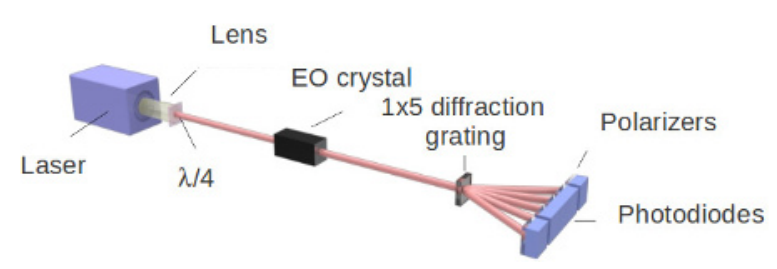

Figure 8. Schematic of the EO sensor (one contact-less electrometer) dedicated to contact-less voltage characterization.

A laser beam is initially collimated thanks to a gradient index lens. The beam crosses the crystal and its polarization state is thus modified due to the E-field induced index modifications of the crystal. A diffraction grating splits the beam in five paths in order to reconstruct the outer modulated polarization, this latter one carrying the transverse E-field vector information [8]. The photodiodes convert finally the EO signal into a photocurrent which is acquired in real time. An example of experimental result is presented in figure 9 . A $220 \mathrm{~V}$, $50 \mathrm{~Hz}$ fed cable (involving three conductors phase shifted of $120^{\circ}$ ) exhibits a surrounded E-field which is measured with the electro-meter.

a)

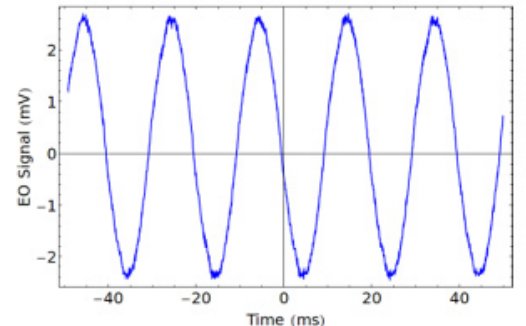

b)
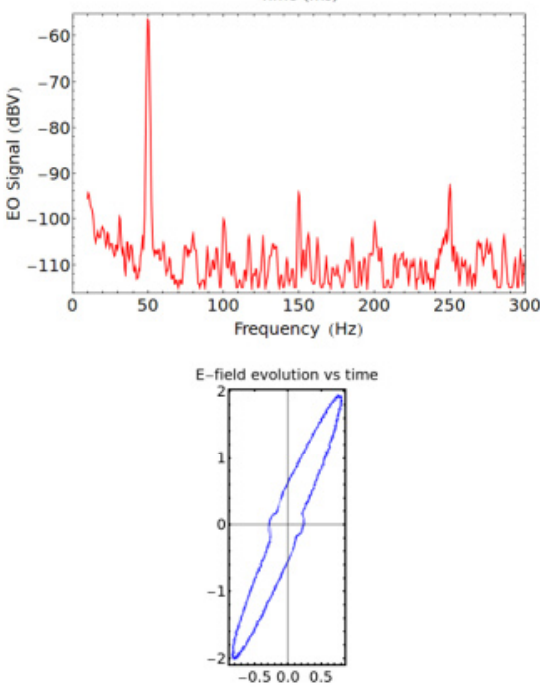

c)

Figure 9. a) Transient signal recorded at the output of one photodiode. b) Fast Fourier Transform of the transient signal. c) Reconstructed transient evolution of the E-field vector obtained with the signal of the five photodiodes.

This result demonstrates the contact-less measurement of the power cable associated E-field. The measurement results show the presence of harmonics (figure 9.b). It has been analytically demonstrated that the E-field evolution describes ellipses depending on the phase, the voltage and the position of each conductors. A reconstructed ellipse is shown on figure 9.c. Nevertheless this single point E-field evolution (with a single electro-meter) is not sufficient to recover the actual voltages carried by the three conductors of the cable. Indeed, four electro-meters are required to measure the phase, the magnitude and the location of each conductor. The electro-meters have to be positioned at equal distance from the centre of the cable. For that purpose, they are implemented within a dielectric ring clamped to the cable. Finally, the link between the four E-fields temporal evolutions (ellipses) and the voltages to be characterized is given by the resolution of an inverse problem. A patent has been deposited on this matter [9].

\subsection{CHIC software power meter for industrial electrical furnace}

To complement the physical approach described above, a software approach was also investigated within the CHIC research project in order to measure power for a particular equipment. The industrial equipment that was chosen is an electrical furnace. Industrial electrical furnaces present the following advantages:

1. power is related to the command signal, which is a value that varies between 0 and $100 \%$,

2. the command signal can easily be collected from the furnace control system.

The system that was studied in represented in the figure below:

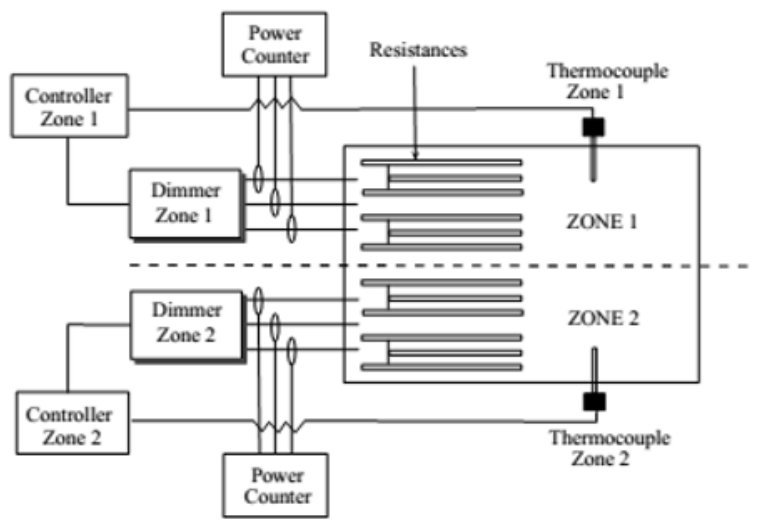

Figure 10. Industrial electrical furnace (with 2 heating zones) that was used to build a software based current sensor.

The figure below presents some of the data collected. We can see that during the first part of the measurements, power is strongly related to the command signal. This is usually the case. When power is not related to the command, as shown in the figure below, it means that there is a problem with the furnace. An overshoot in power may be caused by a door that was poorly closed or by a default in one of the resistances of the furnace (as it is the case here - a set of resistances have been disconnected on purpose). It is very interesting to "see" 
such defaults because this would help sending warnings on the operating conditions of the furnace so as to decrease its consumed energy and to plan for its maintenance.

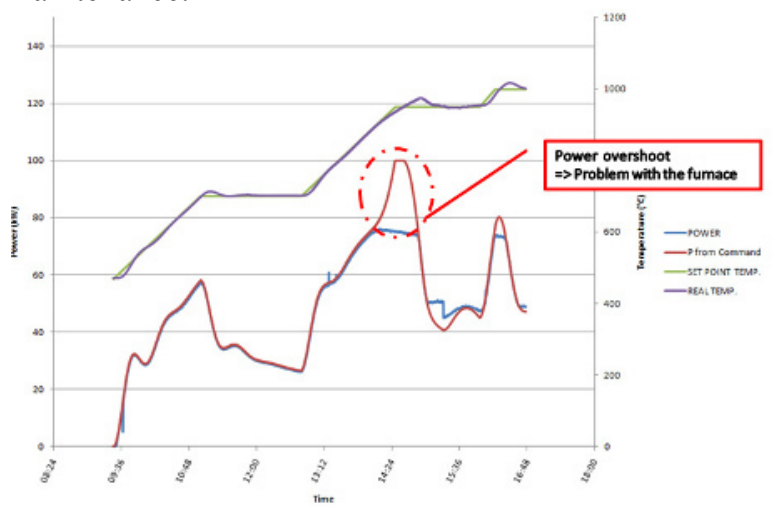

Figure 11. Power, command and inside temperature for one of EDF R\&D electrical furnace

The software power sensor development comes down to model the set dimmering device plus resistances defined by the relationship $P=f(u)$ between the power $P(t)$ and the control signal $u(t)$.

Knowing that the furnace nominal power is of $146 \mathrm{~kW}$ and that the control signal varies between 0 and $100 \%$, the theoretical model of the system is: $\mathrm{P}(\mathrm{t})=$ $1,44 \times u(t)$. As stated above, experimental data reveals such a linearity:

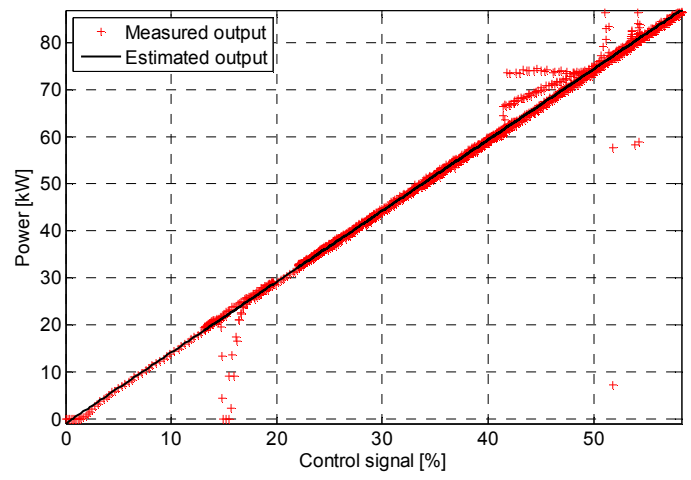

Figure 12. Relationship between Power and control signal for an industrial electrical furnace.

Thus, a simple least-squares algorithm is sufficient to model this relationship and gives $\mathrm{P}(\mathrm{t})=1.506 \times$ $u(t)-0.9117$. The figure above shows both the measured and the simulated values.

The figure below represents a comparison between the theoretical model and the LS-model. The calculation of the relative error taking into account the outlier points, shows that its maximum is less than the fixed objective of $5 \%$ with a clearly advantage for the LS-model.
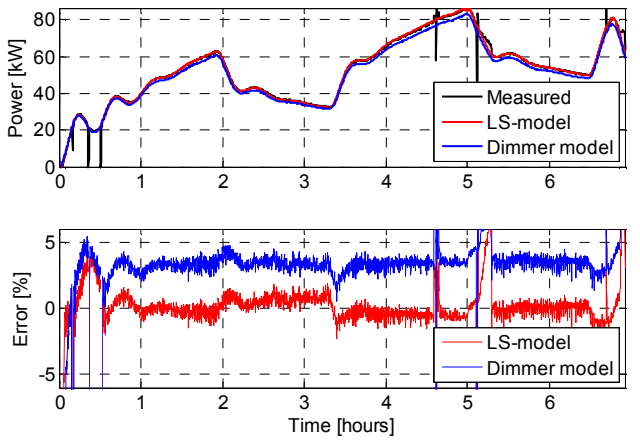

Figure 13. Comparison between the LS-model and the theoretical model during a test heating cycle.

The main advantage of the estimated model is its simplicity. It can be easily implemented within a microcontroller for instance. It also gives the possibility to calibrate or recalibrate the model online.

More details on the ongoing developments can be found in [10].

\section{CHIC flow meters}

\subsection{Costs and benefits for $\mathrm{CHIC}$ flow meters}

CHIC flow meters targeted purchase price has been set to $1300 €$, which is an intermediate value between the purchase costs of the two commercial flow meters presented above. If CHIC flow meters purchase price were too high, they will be rejected as are nowadays the ultrasound flow meters. It is foreseen that CHIC flow meters won't need maintenance, because they are basically software sensors.

CHIC flow meters being non intrusive, they should be accepted fairly widely as long as their price remains within acceptable boundaries. Within the project a particular attention will be devoted to decrease as much as possible the cost of these flow meters.

\subsection{CHIC software flow meter for compressed air systems}

Within the French manufacturing sector, little attention is paid to compressed air systems as long as operators are getting enough air at the correct pressure. Compressed air systems energy consumption is not a priority for plant operation, although many of these systems are wasting energy. A compressed air system is a complex system in which many types of equipment are involved. Compressors play an important role, but dryers, air tanks, pipes, etc. are also to be considered when analysing the system. The figure below shows a typical compressed air system with its main components: 


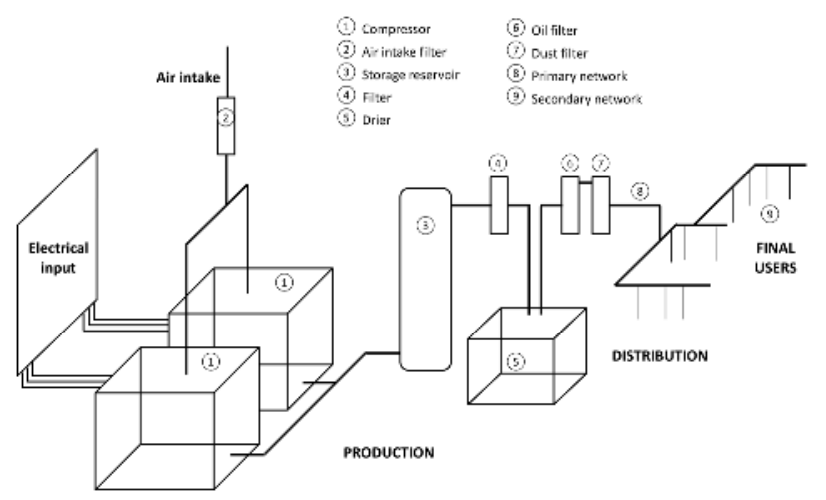

Figure 14. Typical compressed air system with its main components.

The purpose of the CHIC software flow meter for compressed air systems is to estimate the value of the compressed air flow rate produced by a compressor, based on the consumed power of the compressor and on several additional variables such as: the compressed air pressure, the intake air pressure, temperature and humidity.

The EDF R\&D compressed air station used to collect data can be synthesised as follows:

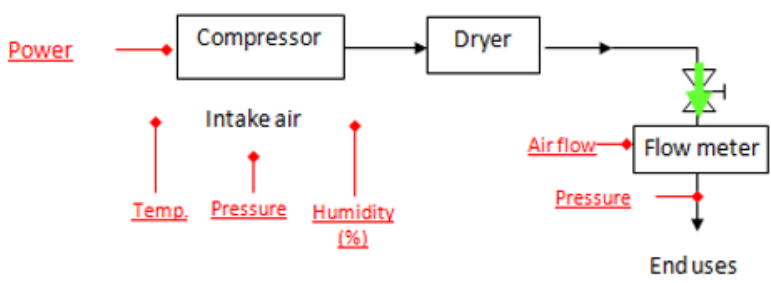

Figure 15. EDF R\&D compressed air system. The data collected are underlined.

CHIC software flow meter for compressed air systems will use the strong correlation that exists between the instantaneous compressor power and the compressed air flow rate - see the left part of the figure below:

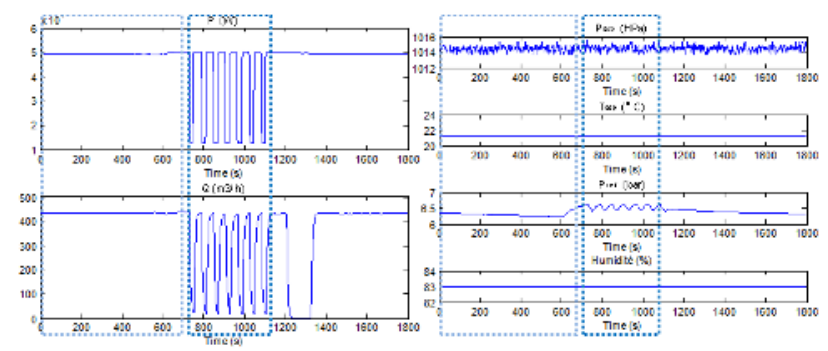

Figure 16. Relation between compressor power and compressed air flow rate (and additional parameters).

Since the compressor is controlled by an on-off valve with respect to the network pressure which is represented in the left part of the figure above, its operating mode is constantly alternating between an idle phase, corresponding to a high network pressure, and a full-load phase, which corresponds to a low network pressure:

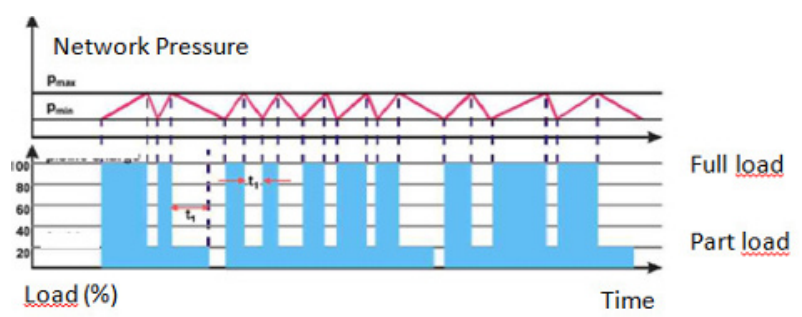

Figure 17. Typical operating conditions for a compressor with no variable speed.

Consequently, the main challenge here is to find a robust mathematical model, able to handle the relation between the controlled power and the air flow rate, depending on influential parameters, such as the network operating pressure and meteorological conditions. The CHIC software flow meter for compressed air systems is based on the following equation:

$Q=\frac{P_{\text {fluid }}}{\frac{k}{k-1} p_{i}\left(\left(\frac{p_{d}}{p_{i}}\right)^{\frac{k-1}{k}}-1\right)}=\frac{r *\left(P-P_{\text {aux }}\right)}{\frac{k}{k-1} p_{i}\left(\left(\frac{p_{d}}{p_{i}}\right)^{\frac{k-1}{k}}-1\right)}$

where :

- $\quad p_{i}, p_{d}$ : intake and discharge air pressures.

- $k$ : polytropic coefficient depending on $p_{i}, p_{d}$, and inlet air temperature and humidity.

- $\quad P, Q$ : respectively represent the electric power consumed and the air flow to be estimated.

- $\quad r$ : compressor efficiency.

- $P_{a u x}$ : Electric power consumed by all the auxiliary equipments.

The three parameters $r, k$ and $P_{a u x}$ need to be identified properly.

The ability of such a sensor to tackle different equipments depends on its calibration. Three ways of calibration are explored within the project:

1. Fixing $k$ to an a priori arbitrary value, taking $P$ and $P_{\text {aux }}$ from the compressor manufacturer datasheet, setting $p_{i}$ to 1 bar (atmospheric pressure), setting the discharge pressure $p_{d}$ to the manufacturer operating point, the air flow to the corresponding flow measured by the manufacturer, and calculating $r$ from the above physical model.

2. Same procedure as the one above, but taking $P$ and $P_{\text {aux }}$ from in-situ measurements (and not from the compressor manufacturer datasheet).

3. Estimating $k$ with respect to the air temperature and humidity, by using the function $k=f$ $\left(T^{\circ}, H \%\right)$ so as to minimize $\Sigma\left(Q_{m e s^{-}} Q_{e s t}\right)$. Such a calibration methodology requires a temporary measurement of the air flow rate (that should be done with a ultra sound flow meter), that may not be possible on several industrial sites. 
The CHIC software flow meter for compressed air systems is not yet operational because in order to derive a robust model, one needs to get data for various operational conditions in terms of pressure, temperature and humidity. Since some operational conditions are weather dependent, we would need roughly an entire year to collect enough data. Work is progressing and a first prototype should be operational pretty soon.

\subsection{CHIC software gas flow meter for boilers}

It is usually very difficult to know the gas consumption of a given industrial gas boiler, since most of the time very few gas meters are installed on industrial sites and the ones that are installed usually measure the site total gas consumption. Once a gas boiler is operational, it is very difficult to convince a plant manager to install a dedicated gas meter because this would cost money and this would imply shutting it down. As a consequence, there is a need for low cost and non intrusive gas flow meters for boilers.

The figure below represents a typical gas boiler. Gas that enters a boiler passes through a regulating valve. It is fairly easy to collect the valve command signal (which is a value that varies between 0 and $100 \%$ ). CHIC gas flow rate software meter will use the intake gas regulating valve command signal in order to derive the amount of gas that is consumed by the boiler.

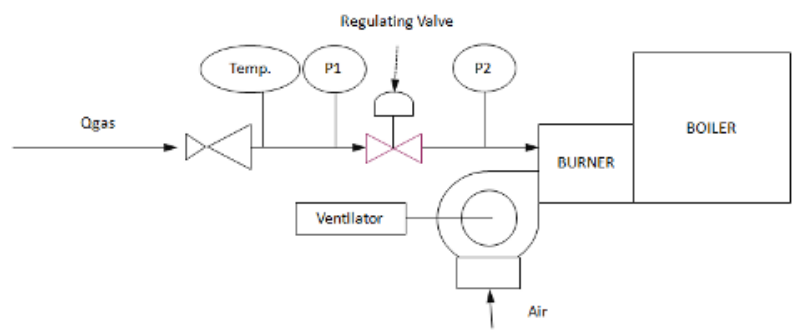

Figure 18. Typical industrial gas boiler installation

The nominal power of gas boiler studied within the project is of $750 \mathrm{~kW}$. The data collected correspond to a stepwise rise of the control $C$ of the regulating valve. Data was collected for different values of gas pressure and temperature. The gas pressure is a manually controlled value but for modelling purposes it is considered as an input. As for the gas temperature, it depends on meteorological conditions.

Two modelling approaches were explored: a black box approach and a grey box approach.

The black box approach consists in estimating the parameters of a parametric model. These parameters depend on the gas pressure and temperature, which finally leads to estimate a Linear Parameter Varying (LPV) model. This model was deduced from the data collected with an electrical valve working at different pressures comprised between 80 and $280 \mathrm{mbar}$ and temperatures between 14 and $32^{\circ} \mathrm{C}$. It has been shown that the temperature have a negligible influence compared to the pressure.
Typical measured signals are presented in the following figure:
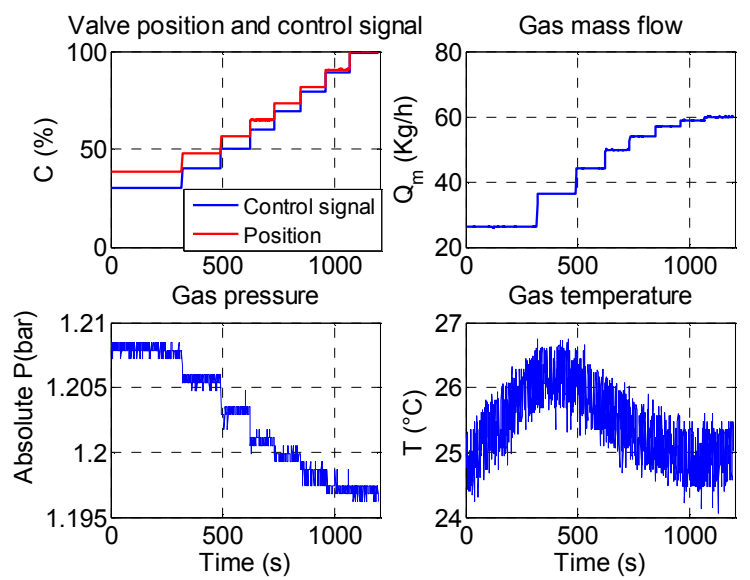

Figure 19. Measured signals for an electronic cylindrical plug valve used at $200 \mathrm{mbar}$

Models are estimated at different setpoints defined by fixed pressures and temperatures. If we analyse the evolution of the gas mass flow rate as a function of the control signal (see figure below), a formulation such as $Q_{m}=\theta_{1} C(t)^{2}+\theta_{2} C(t)+\theta_{3}$ can be deduced and chosen as a local model.

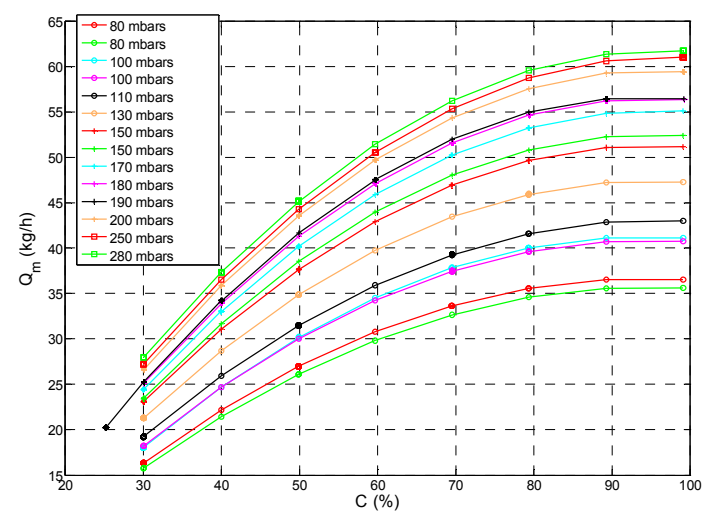

Figure 20. Gas mass flow rate versus control data for different gas pressures.

The evolution of the parameters $\theta_{1}, \theta_{2}$ et $\theta_{3}$ with respect to a fixed pressure, chosen as being an average value which corresponds to the highest control value $C$, has being studied. This pressure is thus necessarily considered as a configuration variable in order to adapt the model to other installations. 

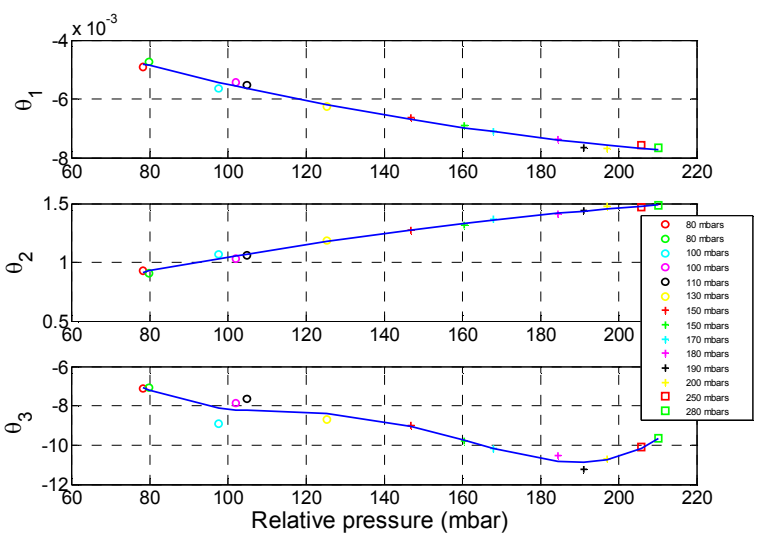

Figure 21. Data collected for $\left(\theta_{1}, \theta_{2}\right.$ et $\left.\theta_{3}\right)$.

From the collected data for $\theta_{1}, \theta_{2}$ et $\theta_{3}$ we derived the following equations for $\theta_{1}, \theta_{2}$ et $\theta_{3}$ :

$$
\left\{\begin{array}{l}
\theta_{1}=\alpha_{1} p^{2}+\alpha_{2} p+\alpha_{3} \\
\theta_{2}=\beta_{1} p^{2}+\beta_{2} p+\beta_{3} \\
\theta_{3}=\gamma_{1} p^{3}+\gamma_{2} p^{2}+\gamma_{3} p+\gamma_{4}
\end{array}\right.
$$

Thus the LPV global model becomes:

$$
\begin{aligned}
Q_{m}(t)=\left(\alpha_{1} p^{2}+\right. & \left.\alpha_{2} p+\alpha_{3}\right) C(t)^{2} \\
& +\left(\beta_{1} p^{2}+\beta_{2} p+\beta_{3}\right) C(t)+\gamma_{1} p^{3} \\
& +\gamma_{2} p^{2}+\gamma_{3} p+\gamma_{4}
\end{aligned}
$$

The simulation results for $200 \mathrm{mbar}$ are presented in the figure below and compared with the measured mass flow. In order to test the CHIC sensor behaviour in real experimental conditions, a "Leave-One-Out" approach was used. Several models were derived using data acquired at (n-1) pressure setpoints and then tested on the remaining $n^{\text {th }}$ pressure stepoint. The results are presented below:
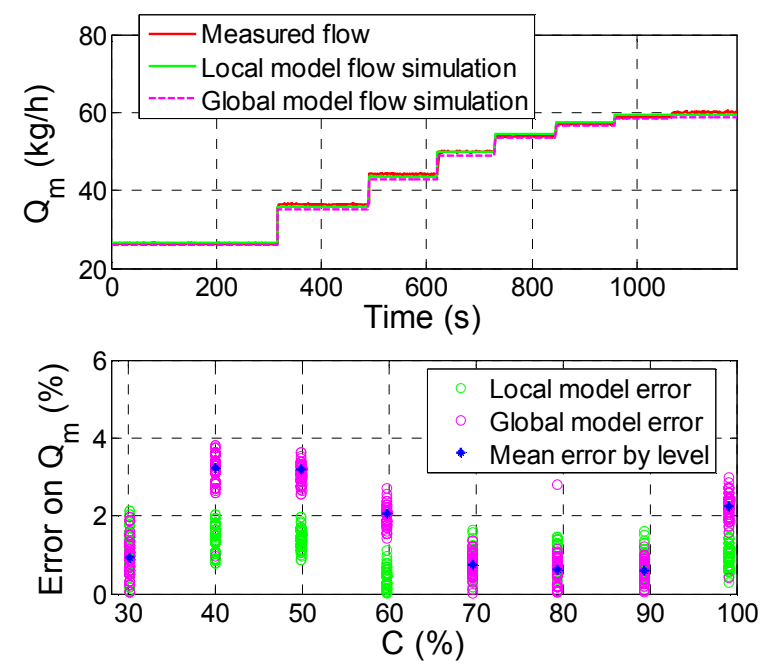

Figure 22. Results for CHIC gas flow rate sensor.

The relative errors of the estimated models are less than $5 \%$, which is satisfactory for sensors dedicated to longterm energy consumption monitoring.

A grey box model approach is also being tested. Its purpose is to find generic models for all sorts of valves existing in Industry for all types of boilers, with different pipe dimensions. There exists a valve opening law: $Q_{\text {gas }}=f(C \%), C$ being the valve control signal and $Q_{\text {gas }}$ the gas flow, which should be different from a valve to another. The equation $Q_{g a s}=K_{v} * \sqrt{p_{1}-p_{2}}$, with $p_{1}$ and $p_{2}$ the pressures before and after the valve, and $K_{v}$ the flow coefficient, needs also to be considered. The objective here is to estimate $Q_{\text {gas }}$ from these two expressions, taking into account the valve manufacturer datasheet and the pipe dimensions. Several methodologies are being investigated.

\section{Conclusion}

The CHIC research project emerged from the continuous awareness that measuring is the key for improving Energy Efficiency in the manufacturing sector and that the installation of power and flow rates measuring devices is still unacceptable for most plant managers, either because this disrupts production of because of its cost. The main purpose of the CHIC project is to develop and test new metering devices that would be non intrusive and low cost so that more and more plant managers will be able to check on their energy consumptions and therefore continuously improve their plant Energy Efficiency.

Two different approaches are explored within the project.

A physical approach is being used to build a clampon power meter for three-conductor cables. We are facing real methodological and technical challenges for this meter because the signals we have to collect and analyse (the values of the magnetic and electrical field around the cable) are very weak and are easily perturbed by the cable environment and operating conditions. Furthermore, these signals need to be synchronised in order to get a value for the electrical power (the value of the $\cos (\phi)$ needs to be computed). A very important simulation work showed encouraging results and two prototypes were built based on these results. These prototypes are to be tested in EDF laboratories to analyse their behaviour in Industry-like operating situations.

A software approach is also investigated within the project. Such an approach is definitely promising, but a lot of work needs to be done in order to get robust models that give satisfactory results for different equipments in many different operating conditions.

\section{Acknowledgement}

The CHIC research project is supported by the French National Research Agency (ANR). 


\section{References}

[1] P. Ripka and M. Janosek, "Advances in Magnetic Field Sensors," Sensors Journal, IEEE, vol. 10, pp. 11081116 (2010).

[2] G. D. R. D'Antona, L. Ottoboni, R. Manara, A., "Processing magnetic sensor array data for AC current measurement in multiconductor systems," Inst. and Meas., IEEE Trans. on, vol. 50, pp. 1289-1295 (2001).

[3] L. Di Rienzo, et al., "Circular arrays of magnetic sensors for current measurement" Inst. and Meas., IEEE Trans. on, vol. 50, pp. 1093-1096 (2001).

[4] X. Chucheng, et al., "An overview of integratable current sensor technologies," in Ind. Appl. Conference, 38th IAS Annual Meeting. Conf. Record of the, pp. 12511258 vol.2 (2003).

[5] M. Bourkeb et al, " Device for measuring currents in the conductors of a sheathed cable of a polyphase network". Int Patent WO2013068360 A1, (2011).

[6] M Bourkeb, O Ondel, C Joubert, L Morel, R Scorretti. "Méthodes numériques pour la mesure de courants dans un système polyphasé", Numelec (2012).

[7] M. Bourkeb, O. Ondel, R. Scorretti, C. Joubert, L. Morel, and H. Yahoui. "Improved AC current measurement approach in multiphase cable using Proper Orthogonal Decomposition" EPJ Appl Phys. (2013).

[8] G. Gaborit, J.-L. Coutaz and L. Duvillaret, "Vectorial electric field measurement using isotropic electro-optic crystals", Appl. Phys. Lett., Vol 90, 24, 241118, 2007

[9] Patent $n^{\circ} 13 / 54984$, reference B12272 FR: « Dispositif de mesure de tension $»$.

[10] Baya Hadid, Erik Etien, Régis Ouvrard, Thierry Poinot, Laurent Lebrusquet, Anne Grau, Gilbert Schmitt. "Soft Sensor Design for Power Measurement and Diagnosis in Electrical Furnace: a Parametric Estimation Approach". To be published in Conference of the IEEE Industrial Electronics Society, IECON (2013).

[11] T. Poinot. "Contribution à l'identification des systèmes par la méthode de surparamétrisation en traitement des eaux". Thesis, Université de Poitiers, France, 1996. 